5. Proof of Example 1.4. If $u$ were continuous, there would exist a compact $K \subset X$ such that $f$ sufficiently small on $K$ implies

$$
|[u(f)](y)|<1
$$

for all $y \in Y$. However, for any such $K$ there exists a $y_{0} \in Y-p(K)$, and if we pick $f \in C(X, R)$ to be 0 on $K$ and 2 on $p^{-1}\left(y_{0}\right)$, then $[u(f)]\left(y_{0}\right)$ $=2$, and hence $f$ fails to satisfy (5.1). This implies that $u$ cannot be continuous.

\title{
REFERENCES
}

1. N. Bourbaki, Espaces vectoriels topologiques, Chapters I and II, Hermann, Paris, 1953.

2. - Espaces vectoriels topologiques, Chapters III and IV, Hermann, Paris, 1955.

3. E. Michael, Topologies on spaces of subsets, Trans. Amer. Math. Soc. 71 (1951), 152-182.

4. - Continuous selections. I, Ann. of Math. (2) 63 (1956), 361-382.

5. - $A$ theorem on semi-continuous set-valued functions, Duke Math. J. 26 (1959), 647-652.

6. - A Short proof of the Arens-Eells embedding theorem, Proc. Amer. Math. Soc. 15 (1964), 415-416.

7. - 1 A linear mapping between function spaces, Proc. Amer. Math. Soc. 15 (1964), 407-409.

UNIVERSITY OF WASHINGTON

\section{A SHORT PROOF OF THE ARENS-EELLS EMBEDDING THEOREM}

E. MICHAEL ${ }^{1}$

In this note, we give a short proof of the following theorem of R. Arens and J. Eells.

THEOREM. Every metric space can be embedded isometrically as a closed, linearly independent subset of a normed linear space.

Proof. Observe first that it suffices if one can always find an isometric, linearly independent embedding. For if $M$ is any metric space, and if its completion $M^{*}$ is thus embedded in a normed linear space $E$, then $M^{*}$ (being complete) is closed in $E$, so that $M$ is closed

Received by the editors February 7, 1963.

${ }^{1}$ Supported by an N.S.F. contract. 
in the subspace $E_{M}$ of $E$ which it spans algebraically (since $\left.M=E_{M} \cap M^{*}\right)$.

So let $X$ be any metric space. Let $Y$ be a metric space (with metric d) which contains $X$ and one point $y_{0} \notin X$. Let $\operatorname{Lip}(Y)$ denote the set of all real-valued functions $f$ on $Y$ such that $f\left(y_{0}\right)=0$ and, for some $K \geqq 0$,

$$
|f(x)-f(y)| \leqq K \mathrm{~d}(x, y), \quad x, y \in Y ;
$$

denote the smallest $K$ which works for such an $f$ by $\|f\|$. This makes $\operatorname{Lip}(Y)$ into a (complete) normed linear space. Let $E$ be its dual, with the usual norm

$$
\|\phi\|=\sup \{|\phi(f)|:\|f\| \leqq 1\} .
$$

Define $h: X \rightarrow E$ by $h(x)=\tilde{x}$, where $\tilde{x}(f)=f(x)$ for all $f \in \operatorname{Lip}(Y) .^{2}$

To see that $h$ is an isometry, let $x_{1}, x_{2} \in X$. Then $\left\|\tilde{x}_{1}-\tilde{x}_{2}\right\| \leqq \mathrm{d}\left(x_{1}, x_{2}\right)$ from the definitions. On the other hand, if $g(y)=\mathrm{d}\left(y, x_{2}\right)-\mathrm{d}\left(y_{0}, x_{2}\right)$ for all $y \in Y$, then $g \in \operatorname{Lip}(Y),\|g\|=1$, and $\left(\tilde{x}_{1}-\tilde{x}_{2}\right)(g)=\mathrm{d}\left(x_{1}, x_{2}\right)$, so that $\left\|\tilde{x}_{1}-\tilde{x}_{2}\right\| \geqq \mathrm{d}\left(x_{1}, x_{2}\right)$.

To see that $h(X)$ is linearly independent in $E$, let $x_{1}, \cdots, x_{n+1}$ be distinct elements of $X$. Then $\bar{x}_{n+1}$ cannot be a linear combination of $\tilde{x}_{1}, \cdots, \tilde{x}_{n}$, for if $g(y)=\mathrm{d}\left(y,\left\{y_{0}, x_{1}, \cdots, x_{n}\right\}\right)$ for $y \in Y$, then $g \in \operatorname{Lip}(Y)$, and $\tilde{x}_{1}, \cdots, \tilde{x}_{n}$ all vanish at $g$ while $\tilde{x}_{n+1}$ does not. That completes the proof.

The author is grateful to V. L. Klee for some helpful conversations.

\section{REFERENCE}

1. R. F. Arens and J. Eells, Jr., On embedding uniform and topological spaces, Pacific J. Math. 6 (1956), 397-403.

UNIVERSITY OF WASHINGTON

2 Although defined quite differently, this embedding seems to be closely related to that constructed in [1]. 